\title{
Use of the gyrotheodolite in underground networks of long high-speed railway tunnels
}

\author{
J. Velasco-Gómez , J. F. Prieto , I. Molina , T. Herrero , J. Fábrega and \\ E. Pérez-Martín
}

The quality of geodetic networks for guiding Tunnel Boring Machines (TBMs) inside long tunnels depends largely on the correct use of a gyroscope. These networks are based on a series of control points at the tunnel entrance, and link each station by means of survey observations as they advance along the tunnel. Once, the networks are used to guide the TBM, they are no longer checked again. It is necessary to perform high accuracy astronomical observations to stars in order to determine the gyrotheodolite constant. Since astronomical observations cannot be made inside tunnels, geodetic azimuths have to be used for the computations. However, these azimuths cannot theoretically be compared with the astronomical azimuths obtained by the gyrotheodolite. An alternative is to compute the instrument constant using the values of the deviation of the vertical derived from a geoid model. That is the approach used in this work where a methodology for the design of underground networks in long tunnels is also presented. This procedure has been implemented during the construction of the Guadarrama and Pajares high-speed railway tunnels (Spain).

Keywords: Gyrotheodolite, Tunnels, Geoid, Deviation of the vertical

\section{Introduction}

The main aim, given the increasing need for long tunnels, is to analyse - as a function of the tunnel length the need for the use of a gyrotheodolite to guide the Tunnel Boring Machines (TBMs), and to determine the constant of this instrument based on the values for the deviation of the vertical obtained from a geoid model.

From the tunnels excavated for the hydraulic works of antiquity to the high-speed rail networks of the present day, one of the most important dangers inherent to this type of project is the possibility of an error in the tunnel alignment, particularly when boring from two portals (Stiros, 2009a). There is currently an increasing demand for long tunnels for high-speed rail networks. One of reasons in favour of the construction of these linear infrastructures is their low environmental impact (Priego, 2009). The geometric characteristics inside the tunnels are highly unfavourable from a geodetic and practical surveying standpoint; therefore, it is essential to ensure a perfect design for the observations (Berberán et al., 2007), the calculation and compensation of the geodetic control networks and for the choice of the appropriate instruments. Furthermore, this network can be used as a guide for the TBMs for the correct breakthrough of the tunnels, as well as for subsequent surveying work. Once the reference geodetic system has been defined for the execution of the project, it is necessary to differentiate between the exterior and the underground part of the tunnel network owing to the peculiarity of this type of work (Velasco et al., 2010). Observations of the exterior network are usually made by means of Global Navigation Satellite Systems (GNSS) techniques, which are less labour-intensive, and more accurate and economical than the so-called classical techniques (Schödlbauer, 1997). In Velasco et al. (2014), we conducted a study of the design, observation, calculation and compensation of such networks. However, the work to optimise the breakthrough of the tunnels focuses fundamentally on the design and observation of the underground networks (Velasco et al., 2015).

The main error inside the tunnel is lateral refraction. The effect of refraction on optical measurements is well known and has been extensively described in literature. Lateral refraction can take place when surfaces of equal of air density are not perpendicular to the plumb-line. Air density mainly depends on atmospheric pressure and temperature. The air temperature inside a tunnel depends not only on the distance to the entrance, but also on the variations in different positions in a tunnel section. Conversely, pressure will generally be constant in the tunnel at the same elevation. Regarding Fig. 1, which shows an idealised situation of a line of sight between two control points inside a tunnel, where the temperature varies on a radial gradient from the tunnel surface to the axis of the tunnel.

The simplified model is based on a distribution of air layers concentric to the axis and uniformly spread in the 


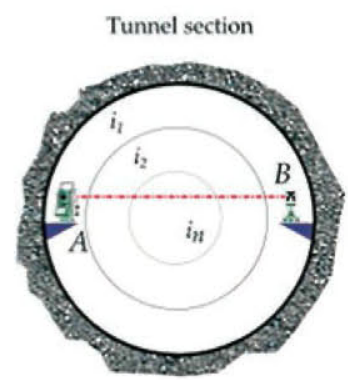

a)

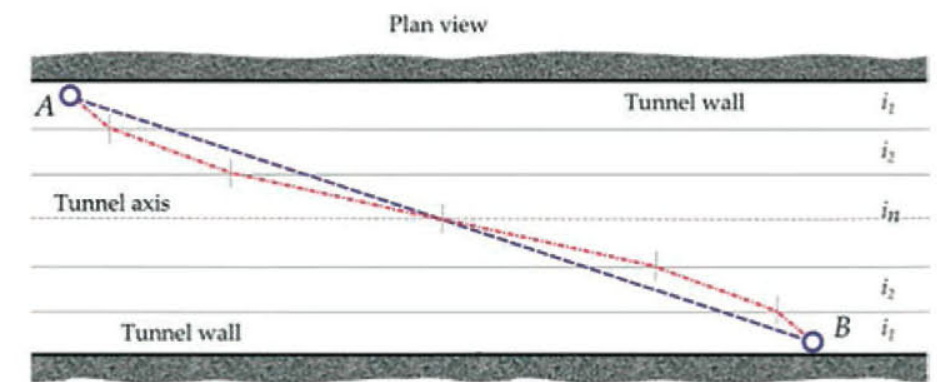

b)
. . . . Theoretical line of sight
Refracted line of sight

1 Effect of lateral refraction between two wall stations. a A distribution of air layers concentric to the axis and uniformly spread in the tunnel direction, and a constant temperature within each layer but with different values, are assumed. $b$ The bold dashed line represents the line of sight from station $A$ to station $B$ free of lateral refraction. The dot-dashed line represents the refracted line of sight forming a 'S-shaped line'

tunnel direction (see Fig. 1a). The temperature is assumed to be constant within each layer but with different values, hence the refraction index will be different. Thus, the variation pattern of the refractive index has an axial symmetry. This temperature gradient varies gradually as we approach the axis of the tunnel. Figure $1 b$ depicts the effect on a theoretical 'line of sight' connecting two stations arranged on either side of the tunnel, i.e. a 'line of sight wall-to-wall'. Both stations are at a similar height in relation to the tunnel axis. Also, these stations are within the same layer of equal refractive index. When the 'line of sight' from station $A$ to station $B$ enters the next layer, and assuming that this new layer has a lower refractive index, the line of sight will suffer a deviation from the normal value to the surface at the crossing point. If the next layer also has a lower refractive index, a new deviation will occur for this line of sight. This effect will be successively repeated for all existing layers inside the tunnel. At this point, the 'optical path' will meet layers whose refractive index will increase at the same rate as it was previously decreasing. So, before reaching the 'tunnel wall' $(B)$, the 'line of sight' will suffer successive deviations in each layer, approaching finally the normal lines to these surfaces at the crossing points between layers. Owing to the fact that the variation of the refractive index has an axial symmetry, the 'line of sight' will suffer a deformation from the axis to the 'tunnel wall' $(B)$, which is symmetric to that initially produced from the 'tunnel wall' $(A)$ to the axis, as can be observed in Fig. $1 b$ forming a 'Sshaped line'.

The most suitable geometry to reduce this effect is a geodetic traverse along the tunnel axis (Chrzanowski, 1981; Johnston, 1991, 1998). Unfortunately, geodetic designs for guiding TBMs need to be integrated into the TBM's chain of execution. Therefore, the measuring of traverses along the tunnel axis is only feasible during stops in construction. Another possibility is to conduct the traverses close to the tunnel walls. Although this geometry follows the tunnel areas with same temperature, it is well known that the existence of excavation dust, humidity and small turbulences owing to the roughness of the excavated surface cause other lateral refraction effects, which are difficult to model (Fowler, 2006). For this reason, geodetic traverses must be designed not along the axis but in a zigzag pattern through the tunnel interior (Korittke, 1990; Hennes,
1998). This wall-to-wall design allows a reasonable compensation of the lateral refraction when measuring the traverse angles (used to carry forward the azimuths), and also enables it to be quantified more easily. Under the commented hypotheses of a practically straight tunnel where the thermal gradients are symmetrical with regard to the axis of the tunnel, we can consider two isolated traverse legs (Fig. 2), made up of three theodolite station positions in $A, B$, and $C$, and analyse the situation in an ideal conditions scenario. The short sides $A-B$ and $B-C$ can be conveniently observed, being the ones used for carrying forward the azimuths. These sides of the traverse are formed by the lines of sight crossing the tunnel wall to wall. $A-C$ side is not observed. As can be seen, considering a straight tunnel, the temperature gradients symmetrical about the tunnel axis, the same traverse leg lengths and a theodolite measuring angles clockwise, the angles between the traverse legs measured by the instruments at points $A$ and $C$ are equally affected by refraction, becoming $A_{\mathrm{r}}$ and $C_{\mathrm{r}}$, which are significantly reduced by the effect of lateral refraction. By contrast, the angle measured at station $B$ is significantly increased by the same amount, becoming $B_{\mathrm{r}}$. This method does not prevent lateral refraction, but ensures

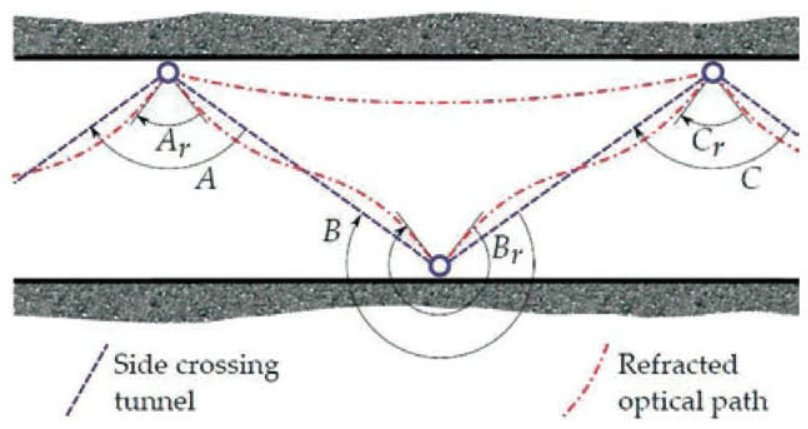

2 Effect of the lateral refraction in a triangle formed by stations $A, B$ and $C$. The bold dashed lines denote the wall-to-wall angle observations. The effect of the lateral refraction is shown by the dash-dotted lines. The angles measured at the stations are respectively $A_{\mathrm{r}}, B_{\mathrm{r}}$ and $C_{\mathrm{r}}$. The angles $A_{r}$ and $C_{r}$. measured at points $A$ and $C$ are equally affected by refraction and are significantly reduced by this effect. By contrast, the angle measured at station $B$ is significantly increased by the same effect (modified from Johnston, 1991) 
that the lateral refraction effects will be largely counterbalanced (Chrzanowski, 1981; Johnston, 1991). The angular misclosure in the triangle $A-B-C$ would allow the quantification of the refraction effect, at least in theory. In practice, side $A-C$ is very close to the tunnel wall and too long, and should be avoided when measuring angles, owing to the unpredictable effects of the above mentioned refraction.

According to Chrzanowski (1981), the use of gyrotheodolites decreases the error propagation owing to the influence of atmospheric refraction. The lateral refraction effect in tunnels has been studied and analysed by Heister (1992), and Heister and Liebl (2010), for example. These studies confirm that this effect can be significantly reduced by using gyrotheodolites. This concept has also been addressed in Ingensand et al. (1998).

When tunnels sections are curved, a zigzag traverse is not as effective in eliminating the problem of lateral refraction, as this effect - as can be seen from Fig. 3 - is more pronounced in tunnel curves (Johnston, 1991). The lines of sight $A-D$ and $B-C$ start very close to the tunnel walls before crossing to the opposite site. However, by running a double zigzag traverse, such effects can be further mitigated (Fowler, 2006). As the observations advance inside the tunnels, measurement uncertainty increases, as can be seen in Fig. 7.

The quality of the observations can be verified by using a gyrotheodolite. Lewén (2006) describes the use of this instrument and its applications in tunnel control networks. In geodesy and surveying, it is used in the form of a gyroscopic theodolite or gyrotheodolite. Its measurements are equivalent to determining astronomical azimuths, since it is always oriented to astronomical North. Brunner and Grillmayer (2002) describe a study of the gyrotheodolite's applications in tunnels, and Korittke and Klapperich (1998) analyse the method of calibrating the gyroscope. The challenge here - based on the instrumentation chosen to design the underground networks in the tunnels - is to determine the geometric design, the length of the network side legs, the number of required observation series, and the length of tunnel at which it becomes necessary to use the gyrotheodolite.

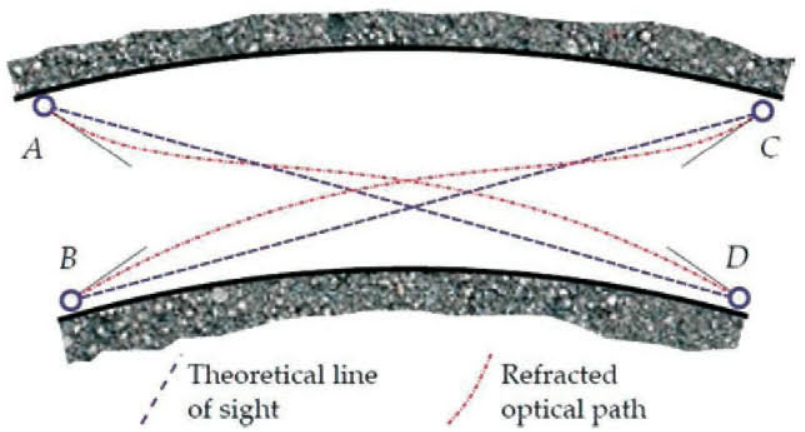

3 Effect of lateral refraction in curved sections (adapted from Johnston, 1991). The bold dashed lines denote the theoretical line of sights whereas the dash-dotted ones represent the refracted lines of sight in this curved section of a tunnel. As almost half of the line of sight runs close to the tunnel walls while the rest is in the central part of the tunnel, the lateral refraction acts differently, and the refracted optical paths are not symmetrical as in the case of Fig. 2
Another challenge is to establish the method for determining the constant for the gyrotheodolite based on a geoid model in the zone. In summary, we need to analyse the optimum conditions for the observations in order to guarantee the guiding of the TBMs and the breakthrough of the tunnels within the required tolerance.

\section{Methodology and simulations}

The methodology described below has been divided into two parts: the first refers to the determination of the gyrotheodolite constant, while the second deals with the design of the underground networks in the tunnels. Finally, this methodology has been used during the drilling of some long tunnels in Spain.

The gyrotheodolite constant is not an adjustment but a mechanical characteristic, being the difference between the gyroscopic north and the astronomical north. The difference between the astronomical azimuth of a line of sight and the reading obtained by observation with a gyrotheodolite gives us the value of this instrumental correction. Its values can be established on the sides of the exterior networks oriented by observations of the Pole star (e.g. $\alpha$-Ursa Minoris, or $\sigma$ Octantis). Once this constant has been determined, it must be corrected, as it is an instant astronomical azimuth referred to the geopotential surface of the observation point. The movement of the pole, altitude of the target point and curvature of the plumb line must all be corrected (Sevilla, 2003; Torge and Müller, 2012). The main problem is that astronomical observations cannot be made inside a tunnel. Thus, the geodetic ellipsoidal azimuths in the interior cannot, basically, be compared to the astronomical azimuths obtained with the gyrotheodolite. The conversion of astronomical azimuths to geodetic ellipsoidal azimuths involves some basic steps. First, instrumental corrections are needed, then, a second correction owing to the deviation of the vertical must be applied. In order to calculate this component, we have to determine the geoid in the work area, or alternatively a geoid model must be used. The influence of the deviation of the vertical has been studied in the Gotthard Base-Tunnel (Carosio and Kanani, 1997). Astronomical observations are a commonly used technique in tunnelling works as they allow an independent control of the GNSS networks, which determine the azimuths at the portals of the tunnels (Solaric et al., 2005). The Laplace equation, which relates astronomical and geodetic ellipsoidal azimuths, is expressed by (Soler et al., 2014)

$$
\begin{aligned}
\Delta A & =A-\alpha=(\Lambda-\lambda) \cdot \sin \phi+(\xi \cdot \sin A-\eta \cdot \cos A) \cdot \cot z \\
\xi & =\Phi-\phi \\
\eta & =(\Lambda-\lambda) \cdot \cos f
\end{aligned}
$$

where $\Lambda$ is the astronomical longitude, $\Phi$ is the astronomical latitude, $\lambda$ and $\varphi$ are the geodetic longitude and latitude respectively, $\xi$ and $\eta$ are the components of the deviation of the vertical and $z$ denotes de zenith angle. Assuming that zenith angles $(z)$ are near $90^{\circ}$, equation (4) is reduced to

$$
\Delta A=A-\alpha=\eta \cdot \tan \phi
$$


The gyrotheodolite constant can be calculated from equation (5), where $\Delta A$ is the difference between the astronomical $(A)$ and the geodetic azimuth $(\alpha)$. The values of the component $\eta$ are determined from the deviation of the vertical on the exterior and the interior of the tunnel at different KP (kilometric points) along it, subsequently analysing the variation in $\eta \cdot \tan \varphi$. If this is less than the nominal precision of the gyrotheodolite, the constant of the instrument can be assumed to be the difference between the geodetic azimuth obtained on the exterior of the tunnel and the gyrotheodolite reading. In other words, this value combines the real constant of the apparatus with the constant caused by the deviation of the vertical. Conversely, if the value exceeds the precision of the instrument, we will need to continue modelling this constant based on the values of the deviation of the vertical in the interior of the tunnel.

Concerning underground networks, this work presents a study carried out by means of simulation techniques using the GEOLAB software (Microsearch Corp.), following the strategy developed in Regidor et al. (2001), in order to analyse the need to use a gyrotheodolite when setting out extra long tunnels.

Considering this experience, different simulations where made in tunnels with these characteristics using zigzag traverses and side lengths of $500,375,250$ and $180 \mathrm{~m}$ (summarised in Fig. 4). This experience and the different simulations led to conclude that the optimum distance was $375 \mathrm{~m}$. Generally, this distance is the greatest at which Leica's Automatic Target Recognition (ATR) equipment can operate, given that the visibility conditions inside a tunnel are far from ideal for observation. Furthermore, the geometry of high-speed rail tunnels permits such side lengths, as the layout has no sharp curves. But designing geodetic traverses with this distance is not sufficient for the daily work of guiding the machine, which must be done whenever it becomes necessary.

That is to say, the apparatus must be changed when it reaches the end of the machine and only at that precise moment, otherwise it will be inaccessible. Thus, the geodetic traverses of this length must be reinforced with intermediate control points, at $180 \mathrm{~m}$ distance from the main ones. Another option is to use geodetic traverses with side lengths of $250 \mathrm{~m}$. Below, we show the simulations made with double crossed geodetic traverses and with a side length of $250 \mathrm{~m}$ (Fig. 5). Since the use of lines of sight wall-to-wall (zigzag) virtually eliminates the lateral refraction error, no corrections to angles were made. The compensation of lateral refraction errors has

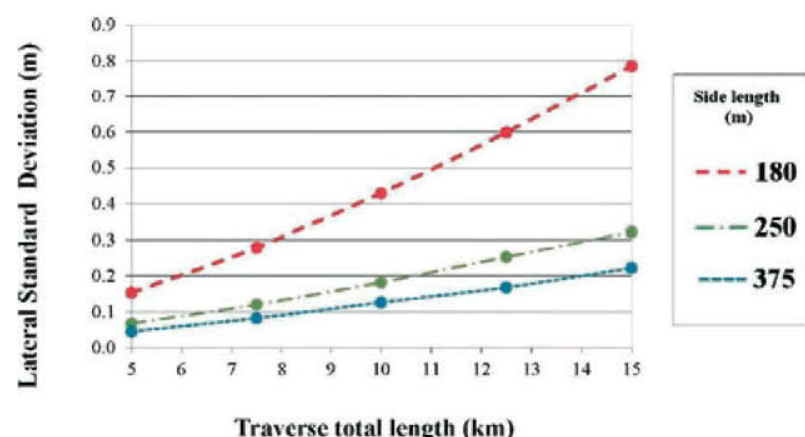

4 Standard deviation propagation for zigzag traverses observed without gyrotheodolite using side length legs of 189,250 and $375 \mathrm{~m}$ been assumed for these simulations. Furthermore, this compensation methodology has been taken into account during field data processing.

Here we define a gyrotheodolite observation as a bearing of each traverse leg. A gyrotheodolite series is defined as a set of direct and reverse gyrotheodolite observations of the traverse leg, as well as a direct and reverse gyrotheodolite observations of its crossed traverse leg at each kilometre point (KP) of the tunnel. Each of the two measured azimuths (direct and reciprocal at a KP) incorporates a lateral refraction error, owing to the S-shaped of the optical path. The two corresponding azimuths observed in the crossed traverse leg at the same KP also incorporate a refractive error but of opposite sense, also owing to the reversed Sshaped of the optical path. This is why the use of the four gyrotheodolite azimuths observed in the two crossed traverse legs, as shown in Fig. 5, will largely eliminate lateral refraction error. Some simulations using these conditions have been performed and are shown in Fig. $7 a$ and $b$.

This procedure was carried out based on the following hypotheses: the precision required in the tunnel breakthrough is $10 \mathrm{~mm}$ per kilometre (Fowler, 2006); the precisions of the instruments used in the simulation were as follows: total station $\pm 0.5^{\prime \prime}$ of nominal precision and $\pm 1 \mathrm{~mm}+1 \mathrm{ppm}$; gyrotheodolite with a nominal precision of $\pm 3^{\prime \prime}$; and instrument set-up and targetting error of $\pm 0.002 \mathrm{~m}$ (centering error). The tunnel is bored from two portals. The observations with the gyrotheodolite are entered as a double crossed azimuth observation set every kilometre point. Figure 6 shows the results for tunnels with a length of $12.5 \mathrm{~km}$ and the uncertainties obtained, revealing that as the number of series made with the gyrotheodolite increases, the number of series made with total station becomes irrelevant. For this purpose, the classical theory of error propagation has been used (Davies et al., 1981; Stiros, 2009b).

Figure $7 a$ shows the $95 \%$ confidence ellipses obtained in the simulation made with side length of $250 \mathrm{~m}, 16$ theodolite angle series and one gyrotheodolite series, Figure $7 b$ shows the simulation results without using the gyrotheodolite. In the first case, the uncertainty is $\pm 0.107 \mathrm{~m}$, which is better than the required precision. The later is $\pm 0.125 \mathrm{~m}$ for a geodetic traverse of $12.5 \mathrm{~km}$, when accepting an error of $1 \mathrm{~cm}$ per $\mathrm{km}$ (Lewén, 2006). In the second case, the uncertainty is $\pm 0.160 \mathrm{~m}$ and, thus, worse than the required precision. Simulations were also made without using the gyrotheodolite by introducing doubled overlapping crossed geodetic traverses with $375 \mathrm{~m}$ side lengths, making 16 theodolite series of angle measurements. The result obtained was $\pm 0.100 \mathrm{~m}$. A tunnel with these characteristics could be surveyed with this method without the use of a gyrotheodolite.

Figure $7 a$ also shows that the measurement uncertainty increases linearly with the use of the gyrotheodolite, whereas the measurement uncertainty increases exponentially in the absence of gyro observations (Fig. $7 b$ ). Table 1 displays a summary of the design of the geodetic traverses obtained from the simulations made without the gyrotheodolite that fall within the tolerances for different tunnel lengths.

\section{Field experiment and results}

Finally, in order to check the results of the simulations, a field campaign was planned and conducted during the 


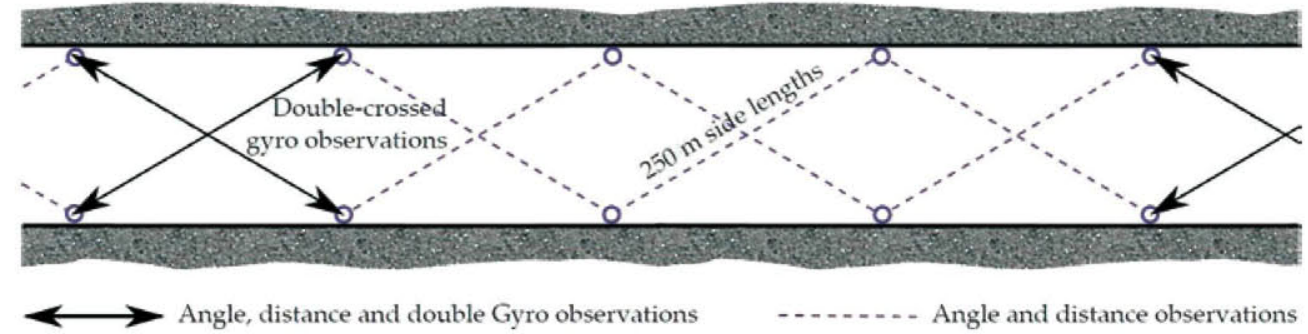

5 Zigzag theodolite and distance traverse with side lengths of $250 \mathrm{~m}$ (dashed lines). The solid arrowed lines represent the double-crossed gyrotheodolite observations. Each double-crossed gyrotheodolite observation consisted of a series of gyrotheodolite determination on each of the four station points

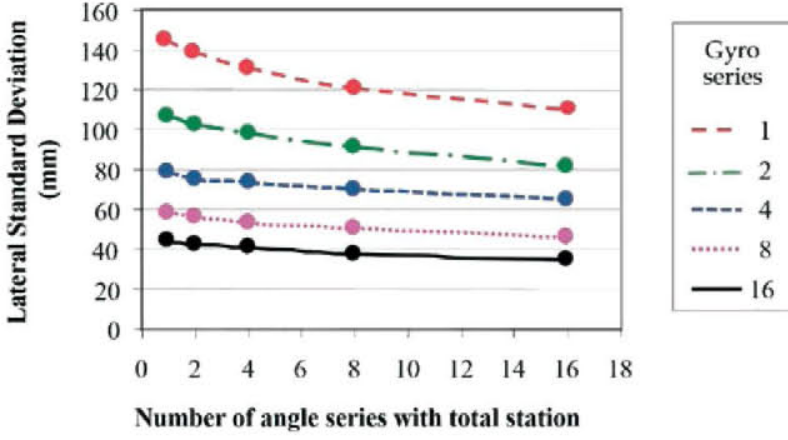

6 Zigzag traverse $(\mathbf{1 2 . 5} \mathrm{km}$ tunnel length, $250 \mathrm{~m}$ side lengths). Lateral standard deviation of the end point at $12.5 \mathrm{~km}$ in function of the number of repeats of total station and gyro measurements

drilling of the tunnels in Pajares. The tunnels are located on the new high-speed railway line giving access to Asturias from the Castilian plateau and crossing the 'Cordillera Cantábrica' mountain range in northern Spain (see Fig. 8).

The tunnels have an approximate length of $25 \mathrm{~km}$ and are formed by two parallel tubes separated $40 \mathrm{~m}$ from each other. They follow an approximate North-South direction. The boring was done from two sides (North and South) with four Tunnel Boring Machines (TBM) and a fifth one on an intermediate central front using an access gallery of $5.5 \mathrm{~km}$, as shown in Fig. 9 .

For the field observations for this campaign, five Leica TCA2003 total stations and a Gyromat 2000 unit, with automatic monitoring, were used. This latter instrument ensured both a maximum nominal precision of $\pm 3^{\prime \prime}$ and automatic data recording (Brunner and Grillmayer, 2002). In the computations, the following instrument precisions were used: total station $\pm 0.5^{\prime \prime}$ and $\pm 1 \mathrm{~mm}+1 \mathrm{ppm}$; and instrument set-up and targetting error of $\pm 0.002 \mathrm{~m}$ (centering error). To measure the gyroscopic azimuths of the sides of the geodetic traverses in the tunnels, the instrument was installed on a fixed metal plates attached to the tunnel walls. A coupling device was designed for the Gyromat 2000 to allow this type of set-up. This method ensures the precision and robustness needed to guarantee the stability and absence of vibrations while the gyroscope is in operation. Figures $10 a$ and $b$ show diagrams of the custom coupling designed and made for the Gyromat 2000.

In order to determine the gyrotheodolite constant as described previously, we used a local geoid model (Sevilla, 2006), and determined the values of $\eta$ by using the Geolab software. Table 2 shows a summary of the values obtained.

As can be seen, the values range between 1.6" and (4.9" at KP 0 and KP 24.2 respectively; there is a difference of $6.5^{\prime \prime}$ along the tunnel. As the nominal precision of the gyrotheodolite is $\pm 3^{\prime \prime}$, the constant can be assumed to be the difference between the geodetic azimuths and the instrument reading obtained from the observations in the exterior network of the tunnels. However, the ideal solution is to correct the constant obtained with the different values for the deviation of the vertical at the various KP in the interior of the tunnel.

Below, we present the results of the North section of the East tunnel, with a length of approximately $10138 \mathrm{~m}$. For this tunnel section, we observed 2156 distances (including the repeated observations per line), a)

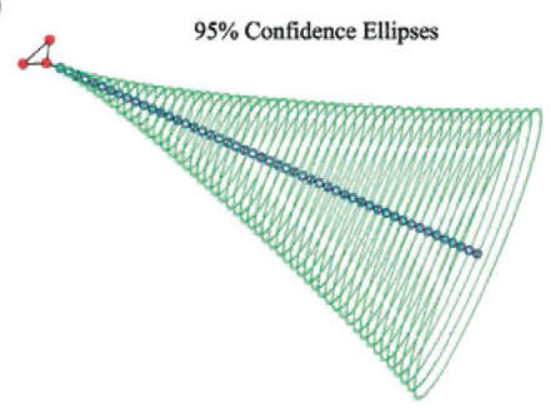

b)

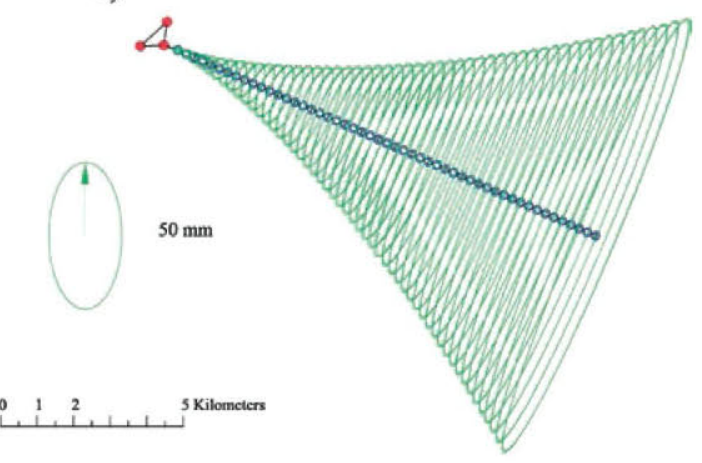

7 a Simulated $95 \%$ confidence ellipses for a zigzag traverse with added gyrotheodolite observation every kilometers ( $250 \mathrm{~m}$ side length, $12.5 \mathrm{~km}$ tunnel length). The scale shows the tunnel length (km) and the ellipse represents the scale for the uncertainty $(\mathrm{mm})$. The control points are shown in the centre of the ellipses. $b$ Simulated $95 \%$ confidence ellipses for a zigzag traverse without the use of the gyrotheodolite ( $250 \mathrm{~m}$ side length, $12.5 \mathrm{~km}$ tunnel length) 
Table 1 Summary of the results for geodetic traverses without gyrotheodolite. Simulation of $5 \mathbf{~ k m}$ tunnel length is computed with $250 \mathrm{~m}$ traverse side length. The simulations for tunnel lengths $7.5 \mathrm{~km}$ and more are computed with two overlapping traverses of 250 and $375 \mathrm{~m}$ side lengths

\begin{tabular}{|c|c|c|c|c|c|}
\hline Geodetic traverse lengths & $5 \mathrm{~km}$ & $7.5 \mathrm{~km}$ & $10 \mathrm{~km}$ & $12.5 \mathrm{~km}$ & $15 \mathrm{~km}$ \\
\hline Traverse side lengths & $250 \mathrm{~m}$ & & & & \\
\hline Doubled traverse side lengths & & $250,375 \mathrm{~m}$ & $250,375 \mathrm{~m}$ & $250,375 \mathrm{~m}$ & $250,375 \mathrm{~m}$ \\
\hline Number of angles & 16 & 16 & 16 & 16 & 16 \\
\hline Lateral uncertainty at $95 \%$ level confidence & $0.047 \mathrm{~m}$ & $0.054 \mathrm{~m}$ & $0.073 \mathrm{~m}$ & $0.114 \mathrm{~m}$ & $0.130 \mathrm{~m}$ \\
\hline
\end{tabular}
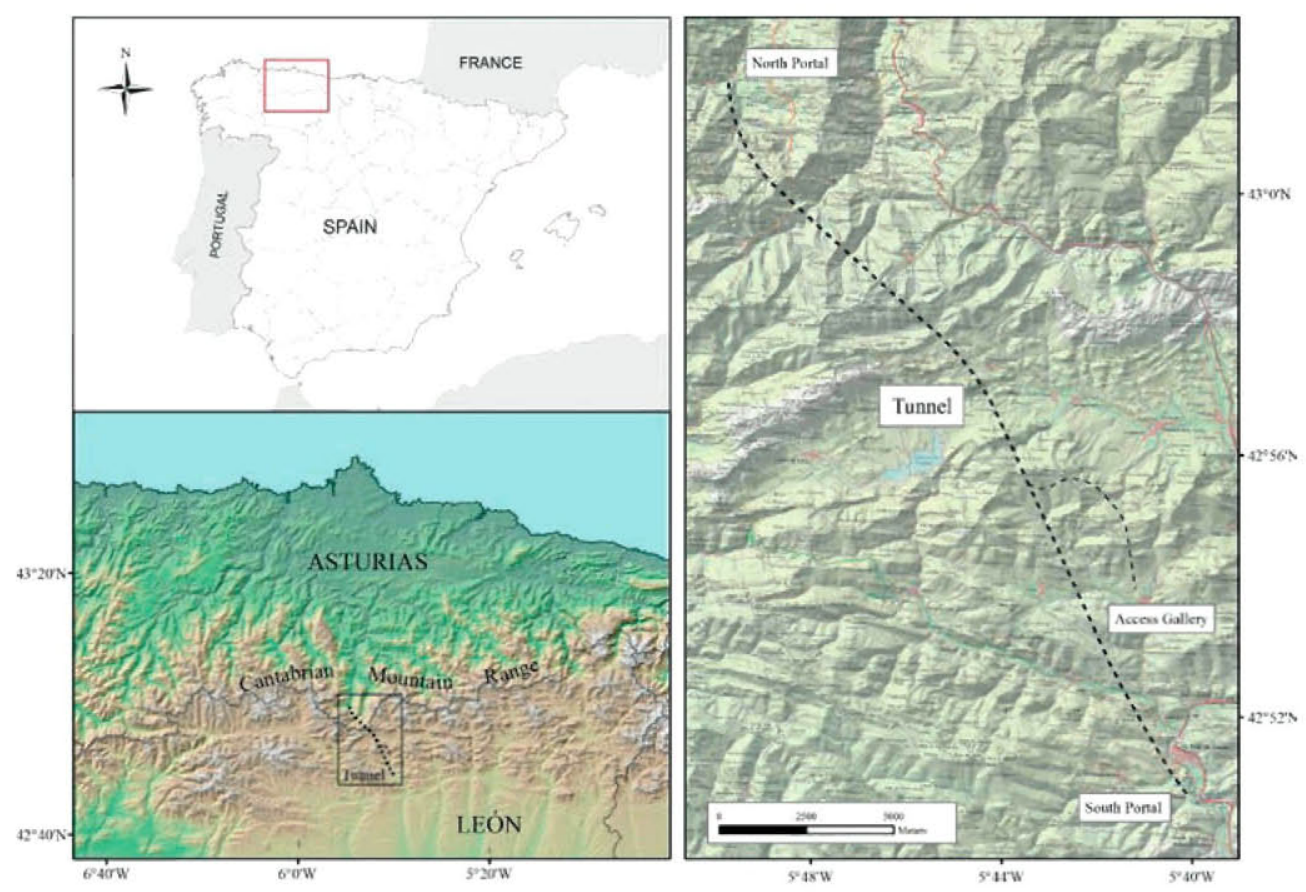

8 Locality of the tunnels used to test the methodology. The high speed railway tunnels of Pajares are located in the 'Cordillera Cantábrica' mountain range in northern Spain. The tunnels are more than $\mathbf{2 4}$ km long with an approximate North-South direction. Also includes a central access gallery to improve the boring procedure

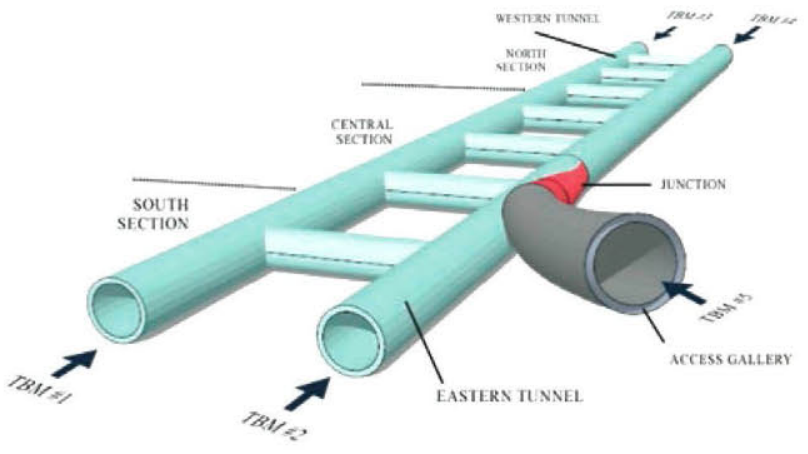

9 Basic sketch of the tunnels in Pajares. The project consisted of two parallel tubes (East Tunnel and West Tunnel) of more than $24 \mathrm{~km}$ length in an approximate North-South direction. Four TBMs started at each of the tunnel portals. In order to accelerate the drilling of the tunnels, a fifth TBM was installed in the central part of the tunnels through an access gallery. This access gallery was excavated from this fifth portal (Buiza portal). The fifth TBM joined the western tunnel and bored the central section

992 theodolite angles (including the repeated observations per station) and 24 gyrotheodolite azimuths (four at each double-crossed gyrotheodolite observation per $\mathrm{km}$ ), and obtained a horizontal uncertainty of $\pm 51 \mathrm{~mm}$ with $95 \%$ confidence at the end point. When eliminating the gyrotheodolite observations, a horizontal uncertainty at $95 \%$ level of confidence of $\pm 122 \mathrm{~mm}$ resulted, this being beyond the required tolerance. Various computations were made by eliminating observations of angles and distances. The results obtained with four angle series per station, two distance measurements per line and one gyrotheodolite measurement per kilometers were $\pm 41 \mathrm{~mm}$ of horizontal uncertainty at $95 \%$ confidence, as well as the elimination of $88 \%$ of the distances and $51 \%$ of the angles observed. Figure 11 shows the $95 \%$ confidence ellipses for this section of tunnel. It includes also the residual lateral refraction error.

The geometry of high-speed railway tunnels is necessarily close to a straight line. This geometry is highly unfavourable from the geodetic standpoint, when one imagines a rectangle of $10 \mathrm{~km}$ by $0.040 \mathrm{~km}$. Underground networks can be observed with sides of equal lengths, which favours their design. However, in certain cases - as below, where for technical reasons it was necessary to make an access gallery to link with the North and South branches - the layout may not be straight, as it does is not have to comply with the design characteristics of a high-speed railway.

The unfavourable geometry of this gallery (in some sections it has curves with a radius of $600 \mathrm{~m}$ ) required particular care both when designing the observations and in computing and adjusting the underground 

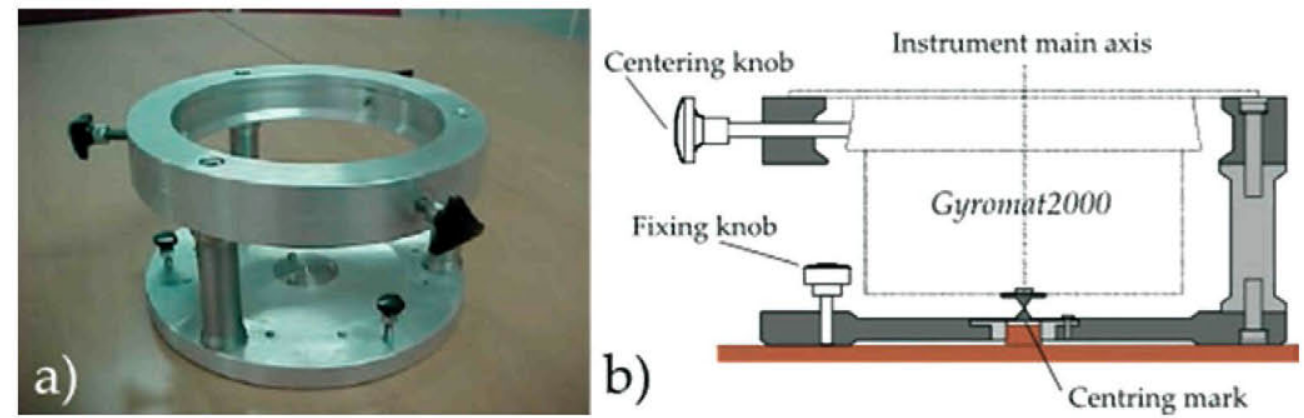

10 a Custom built Gyromat 2000 coupling device designed to guarantee a high centring accuracy during the set-up of the instrument on the control points. $b$ The device consists of a circular base to set on the control point. A top circular ring, where the body of the instrument Gyromat2000 rests, is joined to the base by means of three vertical pillars. Coinciding with the main axis of the instrument and in its lower part, a conical centring witness has been added. Also a similar conical centring witness has been added on the centre of the circular base. The operator sets-up the device with the help of the fixing vertical knobs on the control point. Then, the Gyromat2000 is placed on the upper ring of the device. In order to centre it on the control point, the operator vertically aligns the two conical marks. For this operation, the operator manipulates the three centring horizontal knobs to vertically align the two conical marks

Table 2 Calculated values of $\eta \tan \varphi$ using the local geoid model (from Sevilla, 2006)

\begin{tabular}{llll}
\hline $\begin{array}{l}\text { Dist. origin } \\
\text { Metres }\end{array}$ & $\begin{array}{l}\eta \tan \varphi \\
\text { Arc secs. }\end{array}$ & $\begin{array}{l}\text { Dist. origin } \\
\text { Metres }\end{array}$ & $\begin{array}{l}\eta \tan \varphi \\
\text { Arc secs. }\end{array}$ \\
\hline 0 & $1.5^{\prime \prime}$ & 14200 & $-0.3^{\prime \prime}$ \\
2600 & $0.3^{\prime \prime}$ & 18900 & $-1.5^{\prime \prime}$ \\
10500 & $0.6^{\prime \prime}$ & 22600 & $-2.9^{\prime \prime}$ \\
12800 & $0.3^{\prime \prime}$ & 24200 & $-4.9^{\prime \prime}$ \\
\hline
\end{tabular}

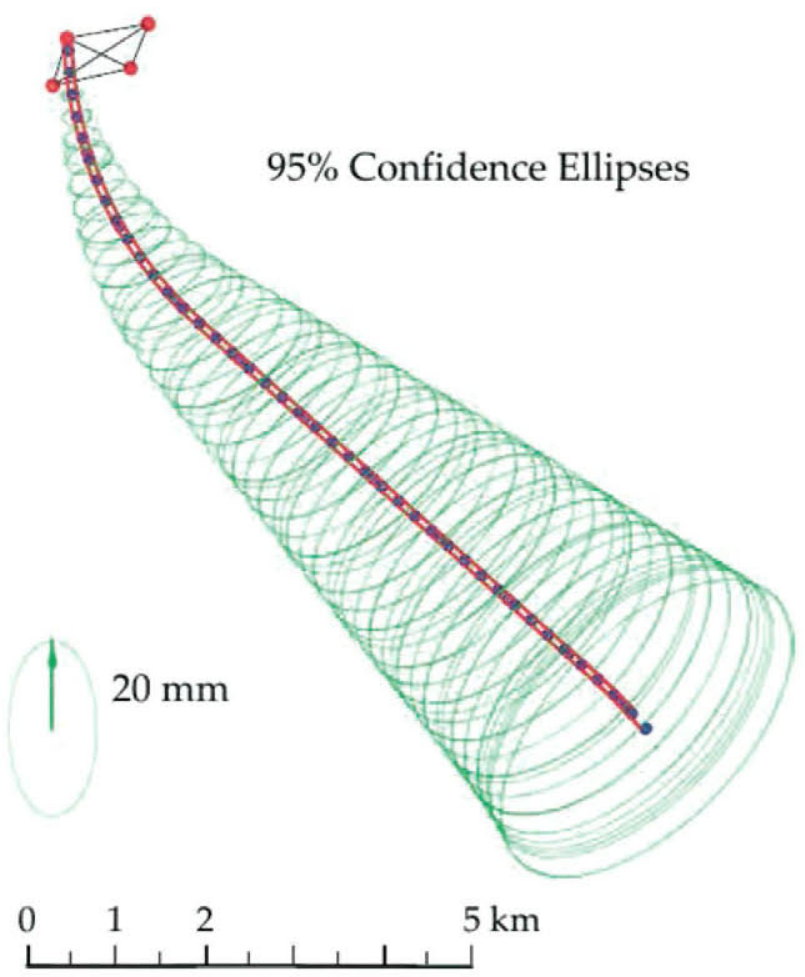

$1195 \%$ confidence ellipses based on actual measurements obtained during the boring of the East tunnel (NorthSouth direction), using gyroscope observations as described on the text. The scale shows the tunnel length $(\mathrm{km})$ and the ellipse represents the scale for the uncertainty $(\mathrm{mm})$

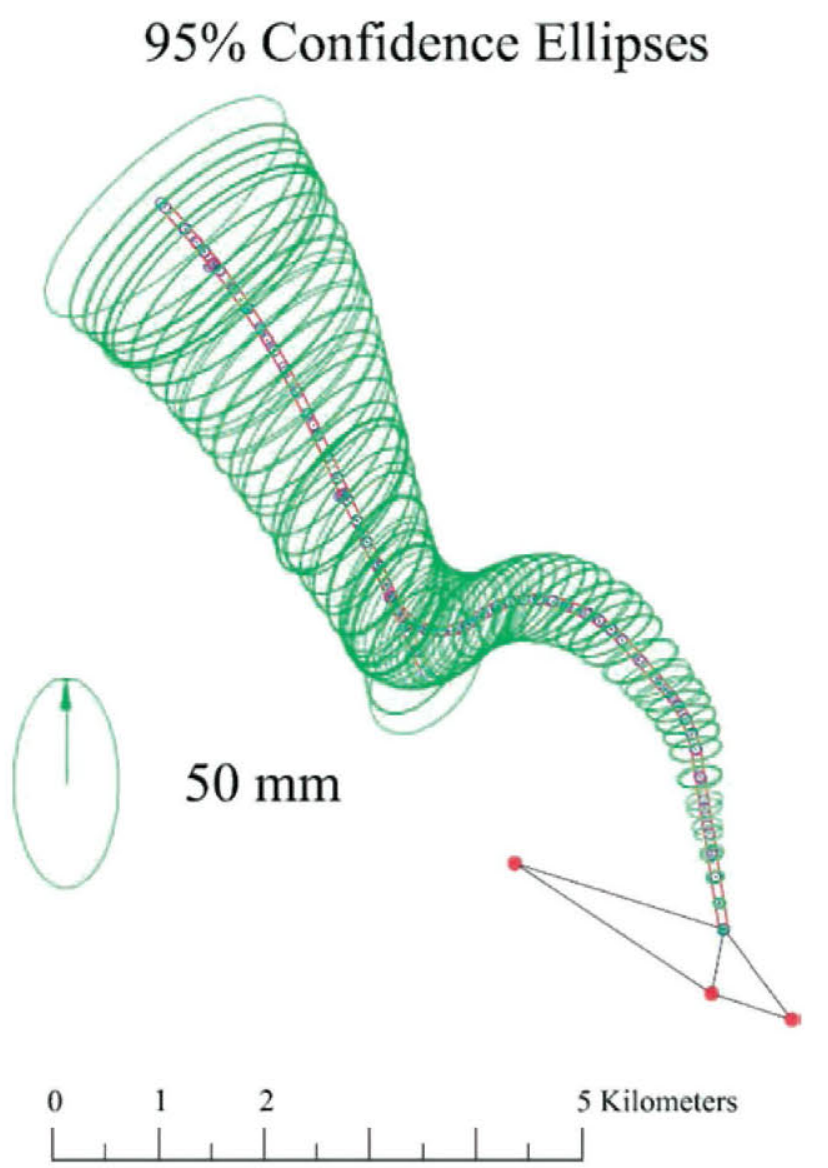

$1295 \%$ confidence ellipses obtained in the network of the fifth tunnel starting at the Buiza portal (central section). The first double curved section is the area of the access gallery while the final straight section links with the main section of the tunnels. The network includes gyroscope observations. The scale shows the tunnel length $(\mathbf{k m})$ and the ellipse represents the scale for the uncertainty (mm)

network. The total length of the access gallery, together with the section of the tunnel linking the North and South branches, was $9815 \mathrm{~m}$. In this section of the tunnel, the use of the gyrotheodolite is essential. For this 


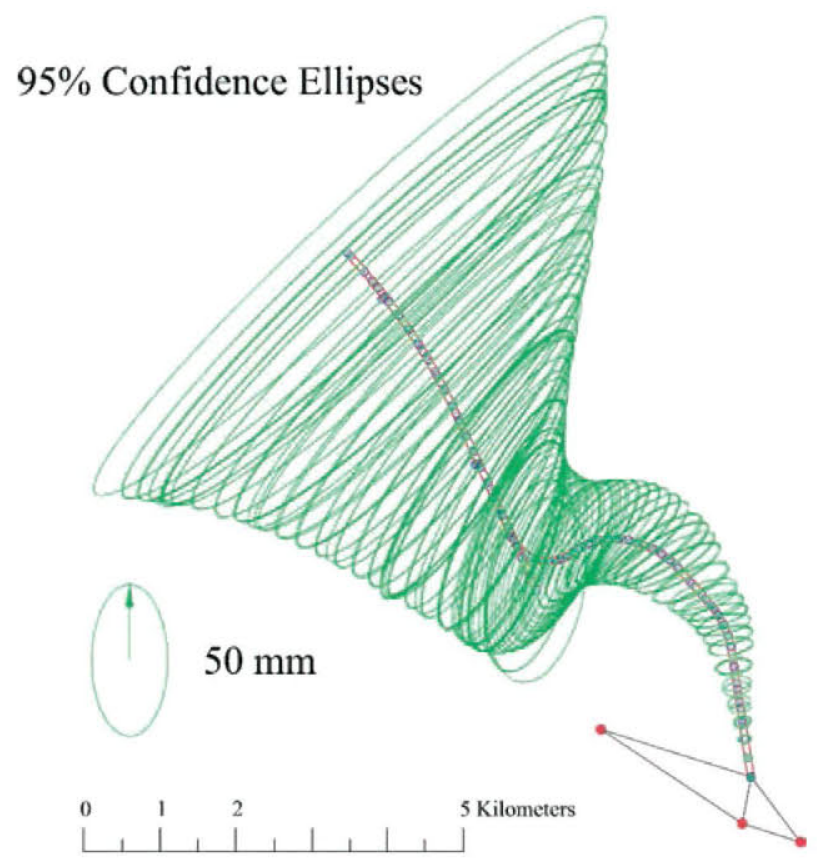

$1395 \%$ confidence ellipses in the central section access gallery (Buiza portal network) without gyrotheodolite data. The scale shows the tunnel length $(\mathrm{km})$ and the ellipse represents the scale for the uncertainty $(\mathrm{mm})$

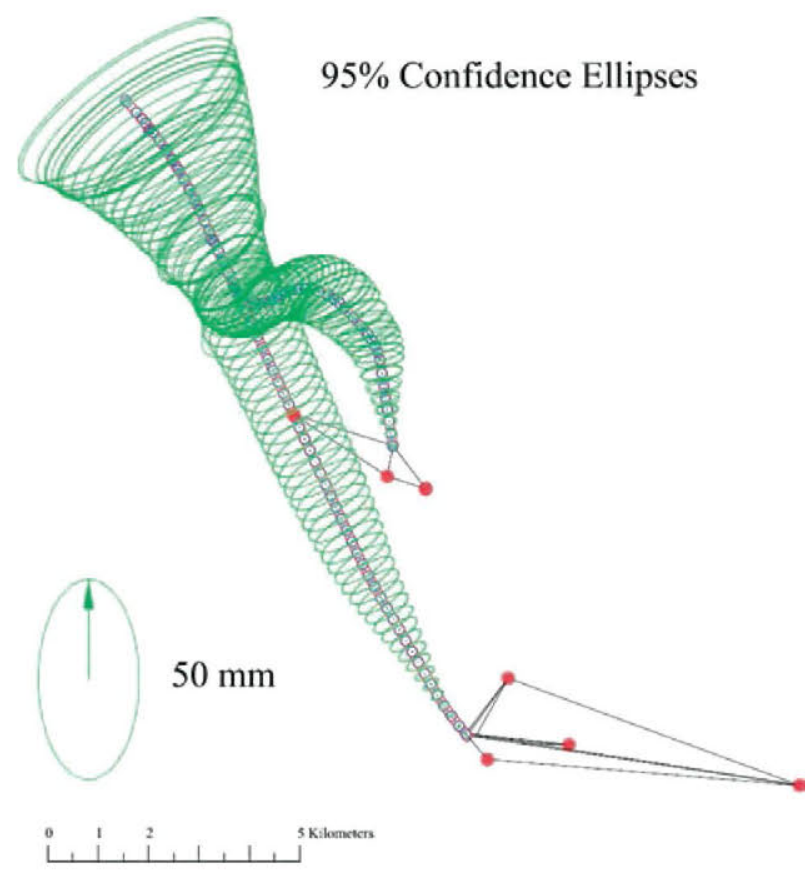

$1495 \%$ confidence ellipses in the junction between the South section and the access gallery of central section. The network includes gyroscope data. The scale shows the tunnel length $(\mathrm{km})$ and the ellipse represents the scale for the uncertainty $(\mathrm{mm})$

tunnel section, we observed 1041 distances, 514 theodolite angles and 38 gyrotheodolite azimuths, obtaining a horizontal uncertainty of $\pm 73 \mathrm{~mm}$ with $95 \%$ confidence at the linking points.

In addition, we calculated the underground network without gyrotheodolite observations, obtaining a horizontal uncertainty at $95 \%$ level of confidence of $241 \mathrm{~mm}$, which far exceeds the tolerance required in the breakthrough. Figure 12 shows the $95 \%$ confidence ellipses achieved with gyroscope observations in this section of tunnel, as well as the geometric design of the entrance gallery. Figure 13 shows the $95 \%$ confidence ellipses obtained without using the gyrotheodolite observations, revealing how the measurement uncertainty increases as a result of the geometry of the layout.

Figure 14 shows the $95 \%$ confidence ellipses obtained in the link between the southern section (drilling northward) and the section corresponding to the access gallery made through the intermediate portal.

\section{Conclusions}

The results obtained with the different simulations lead to the following conclusions:

1. The higher the number of gyrotheodolite series, the lower the influence of the number of angle series.

2. The networks constituted by angles and distances begin at on the exterior networks stations of each portal. As they enter further into the tunnel, the new observations rely solely on observations previously made, which results in the propagation of errors and a lower reliability of the network stations. Networks inside the tunnel can only be verified by using a gyrotheodolite, despite its lower accuracy, 5-8 times lower than that provided by total stations.

3. The outcome of the internal control performed by the gyrotheodolite observations implies that the error propagation, owing to the observations carried out with the total station, must not exceed a certain threshold specified by the manoeuvring capacity of the TBM. Moreover, any systematic error in the initial orientation at the entrance or exit portal will be evident, since the observations from the gyroscopic azimuths have been incorporated.

4. As a consequence of the simulations carried out, the gyrotheodolite usage might be omitted, even though nowadays it is the only survey (geodetic) methodology enabling us to check the quality of the observations before a tunnel breakthrough. However, the use of a gyrotheodolite is considered to be mandatory for tunnels longer than $15 \mathrm{~km}$.

5. Whenever the boring of tunnels with reduced curve radii is required, additional gyrotheodolite observations must be incorporated in order to densify the network. Thus, the length of the geodetic traverse side legs must be shortened.

\section{Acknowledgements}

The authors wish to acknowledge the positive contribution of Pru Brooke-Turner, Dolores Fábregas and Pilar Herranz, who have revised the English translation of the original manuscript. Authors would also like to thank the two anonymous reviewers for their insights and improvements to the final text.

\section{References}

Berberán, A., Machado, M. and Batista, S. 2007. Automatic multitotal station monitoring of a tunnel. Survey Review, 39(305), pp.203-11.

Brunner, F. K. and Grillmayer, E. 2002. On the temperature dependence of gyroscopic measurements using the GYROMAT 2000. FIG XXII 
International Congress. Washington, DC, USA, 19-26 April 2002. Washington: FIG, Available at: < https://www.fig.net/pub/fig_ 2002/TS6-1/TS6_1_brunner_grillmayer.pdf $>$.

Carosio, A. and Kanani, E. 1997. Geodetical methods mathematical models and quality indicators for underground adjustment. FIG-Symposium 1997. Copenhagen, Danemark, 2-5 June 1997. Copenhagen: FIG, pp.153-69.

Chrzanowski, A. 1981. Optimization of the breakthrough accuracy in tunnelling surveys. The Canadian Surveyor, 35(1), pp.5-16.

Davies, R., Foote, F., Anderson, J. and Mikhail, E. 1981. Surveying, theory and practice. 6th edn. New York: McGraw-Hill, pp.992.

Fowler, S. 2006. Design and preanalysis of underground control network for tunnel construction. Dissertation. University of Otago, Dunedin, New Zeland.

Hennes, M. 1998. Zum Einfluss inhomogener Brechungsindexfelder auf die Verschwenkung offener Polygonzüge am Beispiel des Tunnelvortriebs [On the influence of inhomegeneous refractive index fields on the bending of open traverses in tunnels setout networks]. Mitteilung Nr. 65. Zurich: Institut für Geodäsie und Photogrametrie, ETH Zurich, p.88, [in German].

Heister, H. 1992. Zur Anordnung von Krei-selmessungen unter besonderer Berücksichtigung von systematischen Fehlereinflüssen. In: H. J. Matthias and A. Grün, eds. Ingenieurvmessung 92, Beiträgezum XI. Internationalen Kurs für Ingenieurgeodäsie, Bd.1, Ferd. Bonn: Dümmler's Verlag, pp.S.I 7/1-7/14.

Heister, H. and Liebl, W. 2010. Zur Messunsicherheit von Kreiselmessungen im Gothard-Basistunne. Géomatique Suisse, 108(2010), pp.586-93.

Ingensand, H., Ryf, A. and Stengele, R. 1998. The gotthard tunnel - a challenge for geodesy and geotechnics. Symposium on Geodesy for Geotechnical and Structural Engeneerring, ETH, Zurich. April., pp. 20 .

Johnston, A. 1991. Lateral refraction in tunnels. Survey Review, 31(242), pp. 201-20.

Johnston, A. 1998. Tunnel alignment and lateral refraction. Tunnels and Tunnelling International, 30(3), pp.58-60.

Korittke, N. 1990. Influence of Horizontal refraction on traverse measurements in tunnels with small diameter. Proceedings of the Second International Workshop on Accelerator Alignment. Hamburg, Germany, 10-12 September 1990. Hamburg: Deutsches Elektronen Synchrotron DESY.

Korittke, N. and Klapperich, H. 1998. Application of high precision gyrotheodolites in tunnelling. Tunnels and metropolises: proceedings of the World Tunnel Congress'98 on Tunnels and Metropolises. São Paulo, Brazil, 25-30 April 1998. Leiden: Balkema.

Lewén, I. 2006. Use of gyrotheodolite in underground control network, Arkitektur och samhällsbyggnad., PhD. Kungliga Tekniska Högskolan, Sweden.
Priego, E. 2009. Túneles y tuneladoras [Tunnels and Boring Machines]. Valencia, Spain: Universidad Politécnica de Valencia, [in Spanish].

Regidor, J., Prieto, J., Sanz, J. M., Quirós, R. and Barbadillo, A. 2001. El proyecto REGENTE [REGENTE Project]. Topografia $y$ cartografia, 18(105), pp.3-11, [in Spanish].

Schödlbaner, A. 1997. The impact of the global positioning system on tunnel surveying. FIG-Symposium 1997. Copenhagen, Danemark, 2-5 June 1997. Copenhagen: FIG, pp.16-23.

Sevilla, M. J. 2003. Actualización de Cálculos astronómicos [Updating astronomical computations]. Cursos y seminarios del Instituto de Astronomia y Geodesia (CSIC_UCM). 8, pp.84-88., [in Spanish].

Sevilla, M. J. 2006. IBERGEO 2006. Topografia y Cartografía, 23(135), pp.3-20, [in Spanish].

Solaric, N., Versic, Z. and Spoljaruc, D. 2005. Independent control of GPS network above long tunnels astronomically determined azimuths or bearing angles. Survey Review, 39(298), pp.308-16.

Soler, T., Han, J. and Weston, N. 2014. On deflection of the vertical components and their transformations. Journal of Surveying Engineering, 140(2), p.04014005.

Stiros, S. C. $2009 \mathrm{a}$. Orientation and alignment of the 5 th Century BC tunnel of Eupalinus at Samos (Greece). Survey Review, 41(313), pp. $218-25$.

Stiros, S. C. 2009b. Alignment and breakthrough error in tunnelling. Tunelling and Underground Space Techonology, 24, pp. $236-44$.

Torge, W. and Müller, J. 2012. Geodesy. 4th edn. Berlin, Boston: De Gruyter, pp.433.

Velasco, J., Herrero, T., Molina, I., López, J., Pérez-Martín, E. and Prieto, J. 2015. Metodología de diseño, observación y cálculo de redes geodésicas interiores en túneles de ferrocarril de alta velocidad. Methodology for designing, observing and computing of underground geodetic networks of large tunnels for highspeed railways. Informes de la Construcción, 67(538), p.e076, [in Spanish].

Velasco, J., Prieto, J., Herrero, T. and Fábrega, J. 2010. Geodetic network design and strategies followed for drilling a $25 \mathrm{~km}$ tunnel for high-speed railway in Spain. XXIV FIG Congress 2010. Sydney, Australia, 11-16 April 2010. Sydney: FIG, Available at: <http://www.Fig.net/pub/fi2010/papers/ ts06hlts06h_velasco_prieto_et_al_4542.pdf $>$.

Velasco, J., Prieto, J. and Herrero, T. 2014. Metodología de diseño, observación y cálculo de redes geodésicas exteriores para túneles de gran longitud. Methodology for designing, observing and computing of external geodetic networks for long length tunnels. Informes de la Construcción, 66(533), p.e010, [in Spanish]. 